\title{
Fixed Points of Log-Linear Discrete Dynamics
}

\author{
KEN SAWADA ${ }^{a, *}$ and YOSHIO TOGAWA ${ }^{\mathrm{b}}$ \\ ${ }^{a}$ Department of Mathematics, Faculty of Commerce, Waseda University, Tokyo, Japan; ${ }^{b}$ Department of Information Sciences, \\ Faculty of Science and Technology, Science University of Tokyo, Japan
}

(Received 6 December 1996)

\begin{abstract}
In this paper we study the fixed points of the Log-linear discrete dynamics. We show that almost all Log-linear dynamics have at most two fixed points which is a generalization of Soni's result.
\end{abstract}

Keywords: Log-linear discrete dynamics, Fixed points

\section{INTRODUCTION}

The log-linear discrete dynamics

$$
f_{i}\left(x_{1}, \ldots, x_{n}\right)=\frac{c_{i} x_{1}^{a_{i 1}} \cdots x_{n}^{a_{i n}}}{\sum_{j=1}^{n} c_{j} x_{1}^{a_{j 1}} \cdots x_{n}^{a_{j n}}}, \quad i=1, \ldots, n,
$$

have been studied originally as a socio-spacial dynamic model by Dendrinos and Sonis [1]. Many interesting phenomena, for example strange attractors, pitch folk like bifurcations and invariant circles [1-5] have been found to be contained in them.

The log-linear dynamics maps depict a family of dynamics defined systematically by matrix $A=\left(a_{i j}\right)$ and vector $\vec{c}=\left(c_{1}, \ldots, c_{n}\right)^{\mathrm{T}}$; like other such families of dynamics (for instance the Lotka-Volterra dynamics) they are a definitive object of mathematical studies. Therefore a thorough analysis of the log-linear dynamics is necessary because of the importance not only from an applicational view point but also from a pure mathematical view point.

In this paper we investigate the fixed points of the dynamics as our first step of a more extended mathematical study of the log-linear discrete dynamics. We define a real valued function on $R$, which plays a key role in counting the number of the fixed points found in the map, and we prove that almost all dynamics have at most two fixed points. This result is a generalization of Sonis's result [4].

\section{DEFINITIONS AND NOTATIONS}

We begin with some notations and definitions.

For an $n$-dimensional vector $\vec{x}=\left(x_{1}, \ldots, x_{n}\right)^{\mathrm{T}}$, let $(\vec{x})_{i}$ be the $i$ th component of $\vec{x}$, i.e., $(\vec{x})_{i}=x_{i}$.

\footnotetext{
* Corresponding author.
} 
Let

$$
\begin{aligned}
E= & \operatorname{diag}(1, \ldots, 1), \\
& \text { the } n \text { dimensional unit matrix, } \\
\vec{u}= & (1, \ldots, 1)^{\mathrm{T}} \in R^{n}, \\
R^{n+}= & \left\{\vec{x} \in R^{n} \mid x_{i}>0 \text { for } i=1, \ldots, n\right\}, \\
\stackrel{\circ}{ }^{n-1}= & \left\{\vec{x} \in R^{n} \mid \vec{x} \cdot \vec{u}=1, x_{i}>0\right. \\
& \text { for } i=1, \ldots, n\} .
\end{aligned}
$$

For an $n \times n$ matrix $A=\left(a_{i j}\right), \vec{a}_{i}$ denotes the $i$ th column vector of $A$, i.e.,

$$
\vec{a}_{i}=\left(a_{1 i}, \ldots, a_{n i}\right)^{\mathrm{T}}, \quad A=\left(\vec{a}_{1}, \ldots, \vec{a}_{n}\right) .
$$

Given an $n \times n$ matrix $A=\left(a_{i j}\right)$ and $n$ positive real numbers $c_{1}, \ldots, c_{n}$, we define a vector $\vec{\gamma}$ and functions $g_{i}, \vec{g}, g, f_{i}$ and $\vec{f}$ defined on $R^{n+}$ as follows:

$$
\begin{aligned}
\vec{\gamma} & =\left(\log c_{1}, \ldots, \log c_{n}\right)^{\mathrm{T}}, \\
g_{i}(\vec{x}) & =c_{i} x_{1}^{a_{i 1}} \ldots x_{n}^{a_{i n}}, \quad i=1, \ldots, n, \\
\vec{g}(\vec{x}) & =\left(g_{1}(\vec{x}), \ldots, g_{n}(\vec{x})\right)^{\mathrm{T}}, \\
g(\vec{x}) & =\vec{g} \cdot \vec{u}=\sum_{i=1}^{n} g_{i}(\vec{x}), \\
f_{i}(\vec{x}) & =\frac{g_{i}(\vec{x})}{g(\vec{x})} \\
\vec{f}(\vec{x}) & =\left(f_{1}(\vec{x}), \ldots, f_{n}(\vec{x})\right)^{\mathrm{T}} .
\end{aligned}
$$

Since $\vec{f}(\vec{x}) \cdot \vec{u}=1$, the map $\vec{f}$ gives dynamics on the $(n-1)$-simplex $\stackrel{\circ}{\Delta}^{n-1}$.

We call this dynamics the log-linear discrete dynamics.

For a vector $\vec{d}=\left(d_{1}, \ldots, d_{n}\right) \in R^{n}$, let

$$
A[\vec{d}]=\left(\vec{a}_{1}+d_{1} \vec{u}, \ldots, \vec{a}_{n}+d_{n} \vec{u}\right) .
$$

If we modify a matrix $A$ to a matrix $A[\vec{d}]$, then the function $g_{i}(\vec{x})$ becomes

$$
c_{i} x_{1}^{a_{i 1}+d_{1}} \cdots x_{n}^{a_{i n}+d_{n}}=g_{i}(\vec{x}) x_{1}^{d_{1}} \cdots x_{n}^{d_{n}}
$$

and the function $g(\vec{x})$ becomes

$$
g(\vec{x}) x_{1}^{d_{1}} \cdots x_{n}^{d_{n}} .
$$

This implies that the dynamics $\vec{f}$ do not change under the modification $A$ to $A[\vec{d}]$.

Therefore as the canonical form of a matrix $A$, we can consider, for example [1],

$$
\left[\begin{array}{ccc}
0, & \ldots, & 0 \\
*, & \ldots, & * \\
\vdots & & \vdots \\
*, & \ldots, & *
\end{array}\right]
$$

However we will not restrict a matrix $A$ in the canonical form, to keep a free hand for perturbations in the set of $n \times n$ matrices $M(n)$.

Let $V=\{A \in M(n) \mid \operatorname{det}(A-E)=0\}$ and $\tilde{M}(n)=$ $M(n)-V=\{A \in M(n) \mid \operatorname{det}(A-E) \neq 0\}$. Then since $\operatorname{det}(A-E)$ is a polynomial function of $a_{i j}$ 's, $V$ is a $\left(n^{2}-1\right)$-dimensional surface in $n^{2}$-dimensional space $M(n)$. Hence $V$ is a thin set in $M(n)$ and almost all matrices belong to $\widetilde{M}(n)$. Moreover even if $A$ is in $V$, one can modify $A$ to $A[\vec{d}]$ in $\widetilde{M}(n)$ except for the few and rare cases discussed later.

Suppose that $A \in \widetilde{M}(n)$. We define functions of a positive variable $t$ as follows:

$$
\begin{aligned}
\varphi_{i}(t) & =\frac{t^{(B \vec{u})_{i}}}{e^{(B \vec{\gamma})_{i}}}, \quad i=1, \ldots, n, \\
\vec{\varphi}(t) & =\left(\varphi_{1}(t), \ldots, \varphi_{n}(t)\right),
\end{aligned}
$$

and

$$
\Phi(t)=\vec{\varphi}(t) \cdot \vec{u},
$$

where $B=(A-E)^{-1}$. Note that $\Phi(t)$ is not a constant function and that $\vec{\varphi}(t) \in \stackrel{\circ}{\Delta}^{n-1}$ if and only if $\Phi(t)=1$.

\section{FIXED POINTS}

Suppose that $\vec{x}$ is a fixed point of $\vec{f}$, that is $\vec{f}(\vec{x})=\vec{x}$. We can find this fixed point of $\vec{f}$ by 
solving the nonlinear equation system

$$
\begin{aligned}
g_{1}(\vec{x}) & =x_{1} g(\vec{x}), \\
\vdots & \\
g_{n}(\vec{x}) & =x_{n} g(\vec{x}), \\
\vec{x} \cdot \vec{u} & =1 .
\end{aligned}
$$

However we note that it is difficult to solve this nonlinear equation system even numerically.

The following theorem shows that we can find all fixed points of $\vec{f}$ by solving a single nonlinear equation,

$$
\Phi(t)=1, \quad t>0
$$

whose numerical solutions can be easily obtained.

THeOrem 1 Let $A \in \widetilde{M}(n)$. Suppose that the equation $(*)$ has $m$ distinct solutions $t_{1}, \ldots, t_{m}$. Then $\vec{f}$ has just $m$ fixed points $\vec{\varphi}\left(t_{1}\right), \ldots, \vec{\varphi}\left(t_{m}\right)$.

Proof Suppose that $\vec{f}(\vec{x})=\vec{x}$. Then $g_{i}(\vec{x})=x_{i} t$, $i=1, \ldots, n$, where $t=g(\vec{x})$ i.e.,

$$
c_{i} x_{1}^{a_{i 1}} \cdots x_{n}^{a_{i n}}=x_{i} t, \quad i=1, \ldots, n .
$$

Taking logarithms on both sides, we have

$$
\begin{gathered}
\gamma_{i}+\sum_{j} a_{i j} \log x_{j}=\log x_{i}+\log t, \quad i=1, \ldots, n, \\
(A-E)\left(\log x_{1}, \ldots, \log x_{n}\right)^{\mathrm{T}}=-\vec{\gamma}+(\log t) \vec{u} .
\end{gathered}
$$

Since $A-E$ has the inverse matrix $B$, one has

$$
\begin{aligned}
& \left(\log x_{1}, \ldots, \log x_{n}\right)^{\mathrm{T}}=-B \vec{\gamma}+\log t \cdot B \vec{u}, \\
& \log x_{i}=-(B \vec{\gamma})_{i}+\log t \cdot(B \vec{u})_{i}, \quad i=1, \ldots, n .
\end{aligned}
$$

Therefore one obtains

$$
x_{i}=\frac{t^{(B \vec{u})_{i}}}{e^{(B \vec{\gamma})_{i}}}=\varphi_{i}(t), \quad i=1, \ldots, n .
$$

Since $\vec{x} \cdot \vec{u}=1, t$ is a solution of $\Phi(t)=1$.

Conversely we show that if $\hat{t}$ is a solution of the equation $(*)$, then $\vec{\varphi}(\hat{t})$ is a fixed point of $\vec{f}$.
First we notice that $A B=B+E$ since $E=$ $(A-E) B=A B-B$. Then

$$
\begin{aligned}
g_{i}(\varphi(t)) & =c_{i}(\varphi(t))^{a_{i 1}} \cdots\left(\varphi_{n}(t)\right)^{a_{i n}} \\
& =c_{i}\left(\frac{t^{(B \vec{u})_{1}}}{e^{(B \vec{\gamma})_{1}}}\right)^{a_{i 1}} \cdots\left(\frac{t^{(B \vec{u})_{n}}}{e^{(B \vec{\gamma})_{n}}}\right)^{a_{i n}} \\
& =c_{i} \frac{t^{a_{i 1}(B \vec{u})_{1}+\cdots+a_{i n}(B \vec{u})_{n}}}{e^{a_{i 1}(B \vec{\gamma})_{1}+\cdots+a_{i n}(B \vec{\gamma})_{n}}} \\
& =c_{i} \frac{t^{(A B \vec{u})_{i}}}{e^{(A B \vec{\gamma})_{i}}}=c_{i} \frac{t^{(B \vec{u})_{i}+1}}{e^{(B \vec{\gamma})_{i}+\gamma_{i}}} \\
& =t \varphi_{i}(t), \quad i=1, \ldots, n,
\end{aligned}
$$

and

$$
\begin{aligned}
g(\vec{\varphi}(t)) & =\vec{g}(\vec{\varphi}(t)) \cdot \vec{u} \\
& =(t \vec{\varphi}(t)) \cdot \vec{u}=t \Phi(t) .
\end{aligned}
$$

Hence

$$
f_{i}(\vec{\varphi}(t))=\frac{g_{i}(\vec{\varphi}(t))}{g(\vec{\varphi}(t))}=\frac{\varphi_{i}(t)}{\Phi(t)}, \quad i=1, \ldots, n
$$

Therefore if $\hat{t}$ is a solution of the equation, then

$$
f_{i}(\vec{\varphi}(\hat{t}))=\frac{\varphi_{i}(\hat{t})}{\Phi(\hat{t})}=\varphi_{i}(\hat{t}), \quad i=1, \ldots, n,
$$

so that $\vec{\varphi}(\hat{t})$ is a fixed point of $\vec{f}$.

Finally if $\hat{t}$ and $\tilde{t}$ are distinct solutions of the equation, then $\varphi_{1}(\hat{t}) \neq \varphi_{1}(\tilde{t})$ since $\varphi_{1}(t)$ is a monotone function. Hence $\vec{\varphi}\left(t_{1}\right), \ldots, \vec{\varphi}\left(t_{m}\right)$ are distinct.

In Section 5 we give Example 5 in which the coefficients $c_{1}, c_{2}, c_{3}$ are all equal to 1 . Then the equation has no solution. In general:

Proposition 1 Suppose that $A \in \tilde{M}(n)$ and $c_{1}=$ $\cdots=c_{n}=1$. Then the equation $(*)$ has:

1. one solution if $(B \vec{u})_{1}>0, \ldots,(B \vec{u})_{n}>0$,

2. one solution if $(B \vec{u})_{1}<0, \ldots,(B \vec{u})_{n}<0$,

3. no solution if $(B \vec{u})_{i} \geq 0,(B \vec{u})_{j} \leq 0$ for some $1 \leq i, j \leq n$. 
Proof In case (1) (resp. (2)), $\Phi(t)$ is an increasing (resp. decreasing) function and

$\lim _{t \rightarrow+0} \Phi(t)=0($ resp. $\infty), \lim _{t \rightarrow \infty} \Phi(t)=\infty($ resp. 0$)$.

Therefore the equation has unique solution. In case (3)

$\Phi(t)>\varphi_{i}(t)=\frac{t^{(B \vec{u})_{i}}}{e^{(B \vec{\gamma})_{i}}}=t^{(B \vec{u})_{i}} \geq 1 \quad$ for any $t \geq 1$

and

$\Phi(t)>\varphi_{j}(t)=\frac{t^{(B \vec{u})_{j}}}{e^{(B \vec{\gamma})_{j}}}=t^{(B \vec{u})_{j}} \geq 1 \quad$ for any $t<1$

since $\vec{\gamma}=\overrightarrow{0}$. Therefore the equation has no solution.

\section{THE NUMBER OF FIXED POINTS}

In this section we prove that almost all log-linear dynamics have at most two fixed points.

We first prove:

LEMMA 1 Suppose that

$$
h(t)=a_{1} t^{\alpha_{1}}+a_{2} t^{\alpha_{2}}+\cdots+a_{n} t^{\alpha_{n}},
$$

where $\alpha_{1}>\alpha_{2}>\cdots>\alpha_{n}$ and $\alpha_{n}=0$.

(1) If $a_{1}, \ldots, a_{n}>0$, then $h(t)>0$ for all $t>0$.

(2) If $a_{1}, \ldots, a_{k}>0, a_{k+1}, \ldots, a_{n}<0$ for some $k(1 \leq k<n)$, then

$$
\begin{array}{ll}
h(t)<0, & 0<t<t_{0}, \\
h\left(t_{0}\right)=0, & t=t_{0}, \\
h(t)>0, & t_{0}<t
\end{array}
$$

for some $t_{0}>0$.

Proof Note that

$$
\lim _{t \rightarrow \infty} h(t)=\infty
$$

Lemma 1 is true when $n=2$. We may therefore proceed by induction, assuming Lemma 1 true for $n$.
Let

$$
\begin{aligned}
& h(t)=a_{1} t^{\alpha_{1}}+a_{2} t^{\alpha_{2}}+\cdots+a_{n} t^{\alpha_{n}}+a_{n+1} t^{\alpha_{n}+1} \\
& \left(\alpha_{1}>\alpha_{2}>\cdots>\alpha_{n}>\alpha_{n+1}=0\right) .
\end{aligned}
$$

Then

$$
h^{\prime}(t)=a_{1} \alpha_{1} t^{\alpha_{1}-1}+a_{2} \alpha_{2} t^{\alpha_{2}-1}+\cdots+a_{n} \alpha_{n} t^{\alpha_{n}-1}
$$

since $\alpha_{n+1}=0$. We write $h^{\prime}(t)$ in the form

$$
h^{\prime}(t)=t^{\alpha_{n}-1} k(t)
$$

where

$$
\begin{aligned}
k(t) & =b_{1} t^{\beta_{1}}+\cdots+b_{n} t^{\beta_{n}}, \\
b_{1} & =a_{1} \alpha_{1}, \ldots, b_{n}=a_{n} \alpha_{n}, \\
\beta_{1} & =\alpha_{1}-\alpha_{n}, \ldots, \beta_{n}=\alpha_{n}-\alpha_{n}=0 .
\end{aligned}
$$

Note that $\beta_{1}>\beta_{2}>\cdots>\beta_{n}=0$. If $a_{1}, \ldots, a_{n+1}$ $>0$, then $b_{1}, \ldots, b_{n}>0$, so $k(t)>0, t>0$ by the assumption. Since $h(0)=a_{n+1}>0$ and $h^{\prime}(t)>0$ for all $t>0, h(t)>0$ for all $t>0$, so that (1) holds.

If $a_{1}, \ldots, a_{n}>0$ and $a_{n+1}<0$, then $b_{1}, \ldots$, $b_{n}>0$, so $h(0)=a_{n+1}<0$ and $h^{\prime}(t)>0$ for all $t>0$. Since

$$
\lim _{t \rightarrow \infty} h(t)=\infty
$$

there exists $t_{0}>0$ such that:

$$
\begin{array}{ll}
h(t)<0, & 0<t<t_{0}, \\
h\left(t_{0}\right)=0, & t=t_{0}, \\
h(t)>0, & t_{0}<t .
\end{array}
$$

If $a_{1}, \ldots, a_{k}>0$ and $a_{k+1}, \ldots, a_{n+1}<0$ for some $k(1 \leq k<n)$, then $b_{1}, \ldots, b_{k}>0$ and $b_{k+1}, \ldots, b_{n}<0$. Hence there exists $t_{0}>0$ such that:

$$
\begin{array}{ll}
h^{\prime}(t)<0, & 0<t<t_{0}, \\
h^{\prime}\left(t_{0}\right)=0, & t=t_{0}, \\
h^{\prime}(t)>0, & t_{0}<t .
\end{array}
$$


Moreover since $h(0)=a_{n+1}<0, h(t)<0$ for $0<$ $t \leq t_{0}$.

Since $h^{\prime}(t)>0$ for all $t>t_{0}$ and $\lim _{t \rightarrow \infty} h(t)=$ $\infty$, there exits $t_{0}^{\prime}>t_{0}>0$ such that:

$$
\begin{array}{ll}
h(t)<0, & 0<t<t_{0}^{\prime}, \\
h\left(t_{0}\right)=0, & t=t_{0}^{\prime}, \\
h(t)>0, & t_{0}^{\prime}<t .
\end{array}
$$

Therefore (2) holds.

THEOREM 2 Almost all log-linear dynamics have at most two fixed points.

Proof It suffices to show that the equation $(*)$

$$
\Phi(t)=1, \quad t>0
$$

has at most two solutions.

Without the loss of generality, we may write

$$
\Phi(t)=a_{1} t^{\alpha_{1}}+\cdots+a_{l} t^{\alpha_{l}}+\text { const. }
$$

where $a_{1}, \ldots, a_{l}>0$ and $\alpha_{1}>\alpha_{2}>\cdots \alpha_{l}$.

If $\alpha_{1}, \ldots, \alpha_{l}>0(\operatorname{resp} .<0)$, then by the same arguments as the proof of Proposition 1, equation $(*)$ has a unique solution.

Suppose the $\alpha_{1}, \ldots, \alpha_{k}>0$ and $\alpha_{k+1}, \ldots, \alpha_{l}<0$ for some $k(1 \leq k<l)$. Then

$$
\begin{aligned}
\Phi^{\prime}(t) & =a_{1} \alpha_{1} t^{\alpha_{1}-1}+\cdots+a_{l} \alpha_{l} t^{\alpha_{l}-1} \\
& =\alpha_{1} a_{1} t^{\alpha_{l}-1}\left(b_{1} t^{\beta_{1}}+\cdots+b_{l} t^{\beta_{l}}\right),
\end{aligned}
$$

where

$$
\begin{aligned}
& \beta_{1}=\alpha_{1}-\alpha_{l}, \ldots, \beta_{l}=\alpha_{l}-\alpha_{l}, \\
& b_{1}=1, \quad b_{2}=\frac{\alpha_{2} a_{2}}{\alpha_{1} a_{1}}, \ldots, b_{l}=\frac{\alpha_{l} a_{l}}{\alpha_{1} a_{1}} .
\end{aligned}
$$

Note that $\beta_{1}>\cdots>\beta_{l}=0, b_{1}, \ldots, b_{k}>0$ and $b_{k+1}, \ldots, b_{l}<0$. By Lemma 1 , there exist $t_{0}>0$ such that:

$$
\begin{array}{ll}
\Phi^{\prime}(t)<0, & 0<t<t_{0}, \\
\Phi^{\prime}\left(t_{0}\right)=0, & t=t_{0}, \\
\Phi^{\prime}(t)>0, & t_{0}<t .
\end{array}
$$

Therefore $\Phi(t)$ is monotonically decreasing for $t<t_{0}$ and $\Phi(t)$ is monotonically increasing for $t>t_{0}$.

Since

$$
\lim _{t \rightarrow+0} \Phi(t)=\infty, \quad \lim _{t \rightarrow \infty} \Phi(t)=\infty,
$$

the number of solutions is 0,1 or 2 depending on the value of $\Phi\left(t_{0}\right)$. Hence the number of the fixed points is at most two.

Remark We suppose in Theorems 1 and 2 that $A-E$ is invertible. As the coefficients of $A$ are taken randomly, the probability that $A-E$ is noninvertible is zero. However, when the coefficients are restricted to integers, or when one changes an entry of $A$ continuously, one often has to consider a matrix $A$ with $\operatorname{det}(A-E)=0$. So we will study the case $A-E$ when it is noninvertible.

Suppose that $\operatorname{det}(A-E)=0$. In this case one may try to modify $A$ to $A[\vec{d}]$ so that $\operatorname{det}(A[\vec{d}]-$ $E) \neq 0$.

Let $C=\left(c_{i j}\right)=A-E$. Since

$$
\begin{aligned}
\operatorname{det}(A[\vec{d}]-E)= & \operatorname{det}(C[] \vec{d}])=\operatorname{det}(C) \\
& +d_{1} \operatorname{det}\left(\vec{u}, \vec{c}_{2}, \ldots, \vec{c}_{n}\right) \\
& \vdots \\
& +d_{n} \operatorname{det}\left(\vec{c}_{1}, \ldots, \vec{c}_{n-1}, \vec{u}\right)
\end{aligned}
$$

one can choose $\vec{d}$ so that $\operatorname{det}(A[\vec{d}]-E) \neq 0$ except for the case where

$$
\operatorname{det}\left(\vec{u}, \vec{c}_{2}, \ldots, \vec{c}_{n}\right)=\cdots=\operatorname{det}\left(\vec{c}_{1}, \ldots, \vec{c}_{n-1}, \vec{u}\right)=0 .
$$

EXAMPLE Let

$$
A=\left(\begin{array}{lll}
0 & 0 & 0 \\
0 & 2 & 1 \\
1 & 2 & 3
\end{array}\right) .
$$

Then

$$
\operatorname{det}(A-E)=\operatorname{det}\left(\begin{array}{ccc}
-1 & 0 & 0 \\
0 & 1 & 1 \\
1 & 2 & 2
\end{array}\right)=0
$$


and

$$
\begin{aligned}
\operatorname{det}\left(\begin{array}{lll}
1 & 0 & 0 \\
1 & 1 & 1 \\
1 & 2 & 2
\end{array}\right) & =\operatorname{det}\left(\begin{array}{ccc}
-1 & 1 & 0 \\
0 & 1 & 1 \\
1 & 1 & 2
\end{array}\right) \\
& =\operatorname{det}\left(\begin{array}{ccc}
-1 & 0 & 1 \\
0 & 1 & 1 \\
1 & 2 & 1
\end{array}\right)=0
\end{aligned}
$$

So one cannot modify $A$ to $A[\vec{d}]$ with $\operatorname{det}(A[\vec{d}]$ $-E) \neq 0$. For this example, one can get fixed points by simple calculations.

Suppose that $c_{1}=1$. Then $\vec{x}=\left(x_{1}, x_{2}, x_{3}\right)^{\mathrm{T}}$ is a fixed point if and only if

$$
\begin{aligned}
& x_{1}+x_{2}+x_{3}=1, \quad x_{1}, x_{2}, x_{3}>0, \\
& c_{2} x_{1} x_{2} x_{3}=c_{3}\left(x_{1} x_{2} x_{3}\right)^{2}=1
\end{aligned}
$$

This system of equations has no solution except for the case where

$$
c_{3}=c_{2}^{2}, \quad c_{2}>27,
$$

in which case the fixed points make a closed curve in the 2-simplex.

\section{EXAMPLE}

In this section we give some numerical examples illustrating the forms of the function $\Phi(t)$.

Example 1:

$$
\begin{aligned}
c_{1} & =1, \quad c_{2}=1, \quad c_{3}=1, \\
A & =\left(\begin{array}{ccc}
3 & 1 & -1 \\
1 & 1 & 0 \\
-3 & -1 & 3
\end{array}\right) .
\end{aligned}
$$

Then

$$
\Phi(t)=t+t^{2}+t^{3}
$$

is monotonically increasing and the equation has one solution.

Example 2:

$$
\begin{aligned}
& c_{1}=1, \quad c_{2}=1, \quad c_{3}=1, \\
& A=\left(\begin{array}{ccc}
-1 & -1 & 1 \\
-1 & 1 & 0 \\
3 & 1 & -1
\end{array}\right) .
\end{aligned}
$$

Then

$$
\Phi(t)=\frac{1}{t}+\frac{1}{t^{2}}+\frac{1}{t^{3}}
$$

is monotonically decreasing and the equation has one solution.

Example 3:

$$
c_{1}=1, \quad c_{2}=7, \quad c_{3}=50,
$$

$$
A=\left(\begin{array}{ccc}
0 & 1 & -2 \\
3 & 0 & 2.5 \\
2 & 0.5 & 0
\end{array}\right) .
$$

Then

$\Phi(t)=0.209128 t^{0.2}+0.123576 t^{0.488889}+\frac{0.768706}{t^{0.355556}}$

has one minimum $(<1)$ and the equation has two solutions.

Example 4:

$$
\begin{aligned}
& c_{1}=1, \quad c_{2}=7, \quad c_{3}=50, \\
& A=\left(\begin{array}{ccc}
0 & 1 & -2 \\
3 & 0 & -2.57419151135 \\
2 & 0.5 & 0
\end{array}\right) .
\end{aligned}
$$

Then

$$
\Phi(t)=0.209128 t^{0.2}+0.124635 t^{0.500423}+\frac{0.771993}{t^{0.349789}}
$$


has one minimum $(=1)$ and the equation has one solution.

Example 5:

$$
\begin{aligned}
& c_{1}=1, \quad c_{2}=1, \quad c_{3}=1, \\
& A=\left(\begin{array}{ccc}
0 & 1 & -2 \\
3 & 0 & -2.5 \\
2 & 0.5 & 0
\end{array}\right) .
\end{aligned}
$$

Then

$$
\Phi(t)=t^{0.2}+t^{0.488889}+\frac{1}{t^{0.355556}}
$$

has one minimum $(>1)$ and the equation has no solution.

\section{References}

[1] D.S. Dendrinos and M. Sonis, 1990, Chaos and sociospatial dynamics, Applied Mathematical Sciences, Vol. 86, Springer-Verlag.

[2] D.S. Dendrinos, 1993, Entropy oscillations and the discrete spacial relative dynamics map, Socio-Spatial Dynamics 4(1/2), 1-12.

[3] D.S. Dendrinos, 1990, Spatial heterogeneity, relative dynamics, and chaotic behavior, Socio-Spatial Dynamics 4(3), 161-177.

[4] M. Sonis, 1990, Universality of relative socio-spatial dynamics, Socio-Spatial Dynamics 1(3), 179-204.

[5] M. Sonis, 1993, Entropy oscillations and the discrete spacial relative dynamics map: A rejoinder, Socio-Spatial Dynamics 4(1/2), 13-23. 


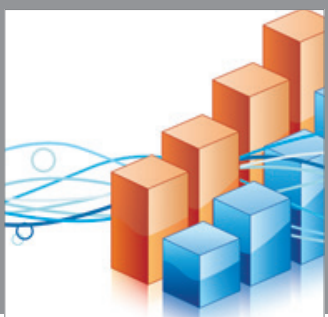

Advances in

Operations Research

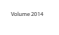

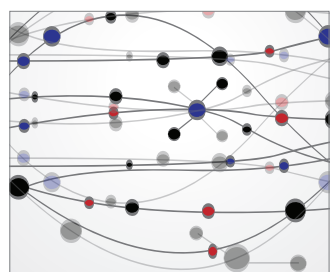

\section{The Scientific} World Journal
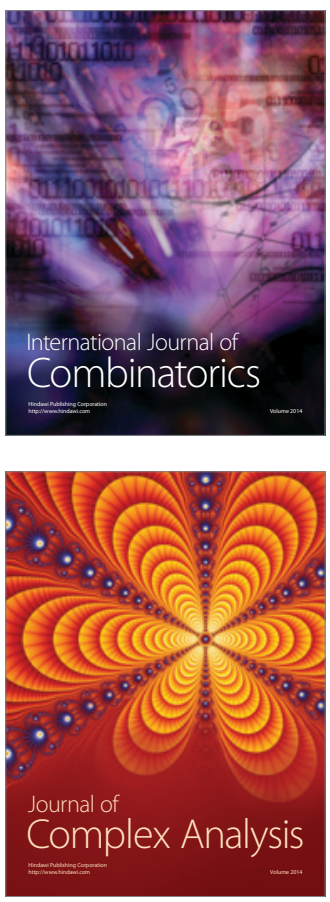

International Journal of

Mathematics and

Mathematical

Sciences
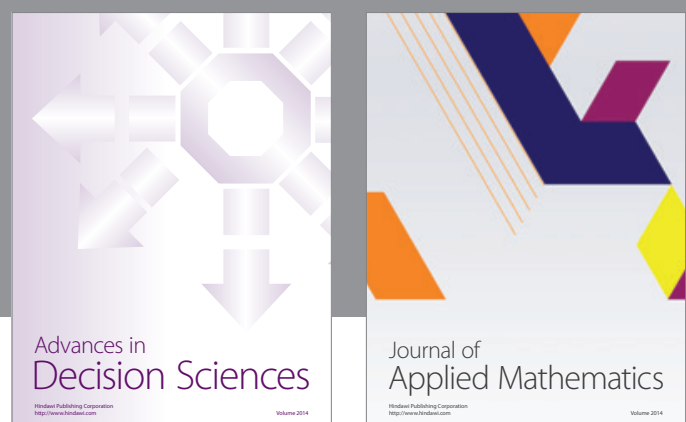

Journal of

Applied Mathematics
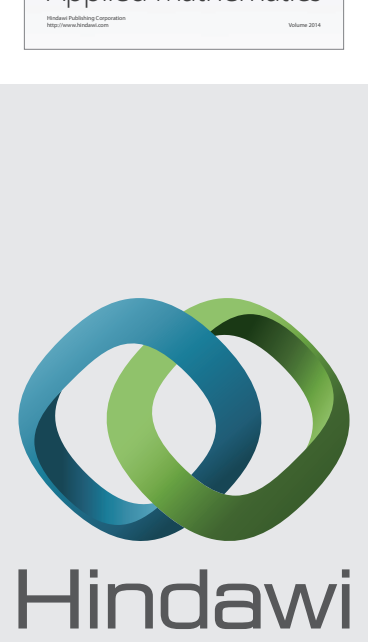

Submit your manuscripts at http://www.hindawi.com
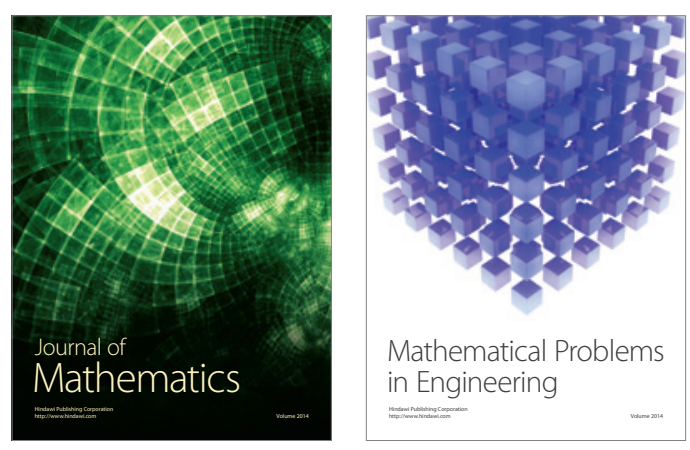

Mathematical Problems in Engineering
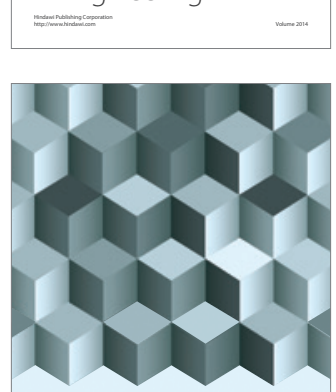

Journal of

Function Spaces
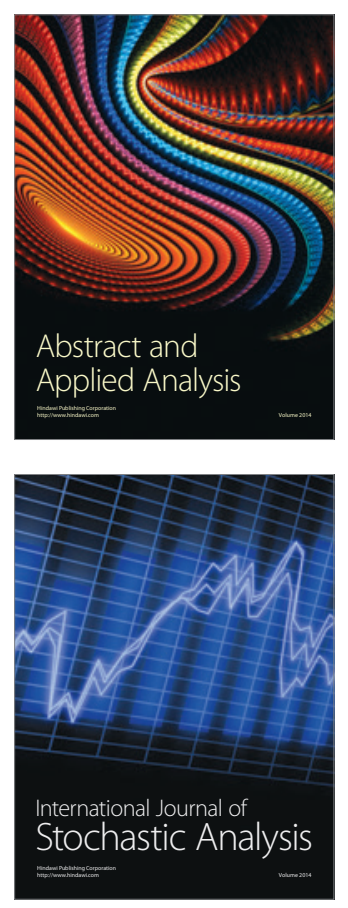

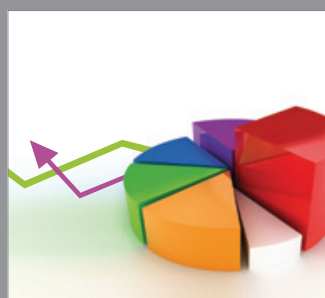

ournal of

Probability and Statistics

Promensencen
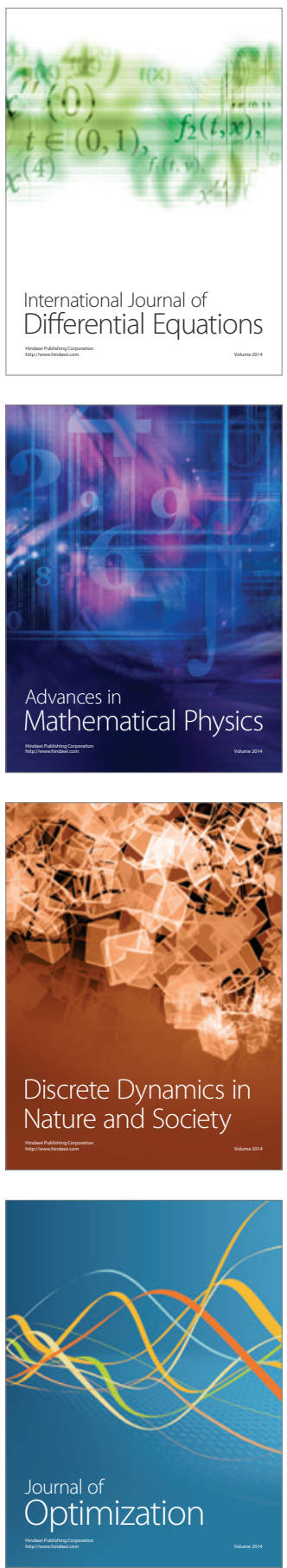